EWA NOWAK-TETER

Political Science Faculty Maria Curie-Sklodowska University, Lublin, Poland

\title{
Review of How to Do Critical Discourse Analysis by David Machin and Andrea Mayr, Sage, Los Angeles 2012, pp. 236, ISBN 978-0-85702-891-4
}

"CDA is an exercise in interpretation, not analysis [...]. CDA is too selective, partial and qualitative [...]. CD analysts [...] are the first to really see and address the workings of power in discourse" [Machin and Mayr 2015, pp. 298-209]. Which of that assertions is true and more grounded than the other is not an easy question to answer. The book of David Machin and Andrea Mayr, at the same time, asks fundamental questions about what is and how to do critical discourse analysis and presents controversies and arguments related to this research approach. The reason for preparing the study was a growing need for explaining and systematizing a set of tools for analysing texts and spoken language funded on a loose combination of approaches rooted in linguistics, but widely applied in media and cultural studies. Studies that use CDA, however, are not only limited to spoken or printed expressions, but also investigate images or video content and other signs of culture like monuments, sounds, toys, pictures, photos, graphics or memes. Especially, the multimodal critical discourse analysis (MCDA) applied in order to investigate the visual communication practices forms the research toolkit that allows for more precise, systematic and careful description and accurate analysis of these practices.

The greatest challenge that CDA is probably able to meet is to reveal the ideology buried in texts and images. By identifying some specific language choices, the critical approach is employed to investigate how language and grammar can be used as ideological instruments, how communication decision are taken when categorizing people, events, places or actions, which persons and phenomena are foregrounded or backgrounded or even excluded, and how certain kinds of practices, ideas and identities are promoted and naturalised. By revealing the language choices, also the 
underlying definitions of events that determine the social actions are possible to be disclosed. Nevertheless, as the authors declare, the CDA is not so much interested in the language use itself, but in the linguistic manifestations of social and cultural processes and structures: "CDA assumes that power relations are discursive. In other words, power is transmitted and practised through discourse" [Machin and Mayr 2015, p. 4].

The study consists of ten parts including the introductory and conclusion chapters (accompanied by Glossary and Index). The first chapter: "Making Active Choices: Language as a Set of Resources", introduces the main theoretical concepts and main principles that form the analytical basis of the MCDA. The chapter is also the first level of expertise the non-linguists researchers would exceed, to be engaged or fascinated with the explanation capacity of the approach. By discussing linguistic determinism and structuralist views, Machin and Mayr present social semiotic theory of communication as being interested in: "[...] the way the communicator uses the semiotic resources available to him, either in language or in visual communication to realise their interests [...]" [Machin and Mayr 2015, p. 17]. They use the example of crime associations to offer the explanation of the social construction of reality:

[...] how we talk about the world influences the society we create [...]. It is our dominant discourse of crime that we end up targeting only the poor and least powerful members of our societies, where we build prisons, use the police in the way that we do, take particular crime prevention measures and vote for political parties that we be tough on crime, rather than creating societies where it is less likely to take place [Machin and Mayr 2015, p. 21].

The subsequent chapters are devoted to the particular means and techniques used in the MCDA in order to investigate texts and images as well as the ideas or values concealed in the linguistic choices. The progress of the analytical process is offered by starting from basic semiotic choices (particularly presenting how the attention to the linguistic and visual details can reveal the implicit meaning) and reaching the more advanced and specific categories of visual and semiotic choices. In particular, the means of presenting speech and speakers (quoting verbs), representing people (language and identity) and representing action (transitivity and verb processes) are studied and illustrated with the relevant examples. The representative strategies are then complemented by the analysis of even more advanced techniques, where, for instance, nominalization and presupposition: "[...] can obscure the agency and responsibility for an action" [Machin and Mayr 2015, p. 137] and are skilful ways by which "[...] authors are able to imply meanings without overtly stating them or present things as taken for granted [...]" [Machin and Mayr 2015, p. 137]. Thereafter, the modality and hedging as well as rhetoric and metaphor are presented as the most precise and sophisticated communication strategies. Using numerous case studies, rhetorical means and metaphors exercised as tools for abstracting process in order to 
reposition the foreground and background are explained. To assess the commitment to the truth, the application of the strategies of modality and hedging is analysed. As there is confirmed, thanks to these features of the language, the subtle signs of people's commitment to what they say can be detected.

The critical approach to the critical discourse analysis is the subject of considerations in the conclusive chapter. The discontents of the CDA and the MCDA include, for instance, a small number of texts or images that $\mathrm{CD}$ analysts are able to involve in; the texts chosen for analysis are presented as typical, although, in fact, they may be not; CDA is too ambitious in its quest for social change; CDA does not allow for systematic comparison between texts. Certainly, as every single method has its own limitations, also critical discourse analysis can be accompanied by complementing approaches. The suggestions made by the critics resulted in offering certain solutions directed to the extending, enriching and making the CDA more rigorous, especially by using corpus studies and ethnographic methods in cooperation with the CDA approach. 